\title{
GeoGebraPrim como instrumento para descubrir y justificar propiedades geométricas en cuarto de primaria ${ }^{1}$
}

\author{
GeoGebraPrim as a tool to identify and justify \\ geometric properties in fourth grade
}

\section{GeoGebraPrim como uma ferramenta para identificar e justificar propriedades geométricas na quarta série}

Recibido: mayo de 2013

Aceptado: agosto de 2013
Alejandro Barbosa ${ }^{2}$

Andrea Escobar ${ }^{3}$

Dra. Leonor Camargo Uribe ${ }^{4}$

\section{Resumen}

Se describe una experiencia de aula en la que el programa GeoGebraPrim desempeña un papel en la introducción a la actividad demostrativa de estudiantes de grado cuarto de primaria, quienes son invitados a construir figuras geométricas, explorarlas en busca de regularidades y justificar algunas propiedades con base en otras. La experiencia se fundamenta en el constructo "actividad demostrativa" del grupo de investigación Aprendizaje y Enseñanza de la Geometría de la Universidad Pedagógica Nacional y en la definición de demostración propuesta por Stylianides (2007).

Palabras clave: Aula; recursos didácticos; recursos informáticos; software; GeoGebraPrim; aprendizaje; procesos cognitivos; procesos de justificación; actividad demostrativa; exploración; conjeturación; justificación.

\begin{abstract}
We describe a classroom experience in which GeoGebraPrim program plays a role in the introduction to the activity of undergraduate demonstrative fourth grade, who are invited to construct geometric figures, exploring in search of regularities and justify some properties based on other. The experience is based on the construct "demonstration activity" research group Learning and Teaching Geometry National Pedagogical University and the definition proposed by Stylianides demo (2007).
\end{abstract}

Keywords: classroom, teaching resources, computing resources, software, GeoGebraPrim; learning cognitive processes, processes of justification; demonstration activity, exploration, conjeturación; justification.

\footnotetext{
1 Artículo de Investigación

2 Universidad Pedagógica Nacional. Bogotá, Colombia. Contacto: mdma_fbarbosa172@pedagogica.edu.co

3 Universidad Pedagógica Nacional. Bogotá, Colombia. Contacto: mdma_jescobar164@pedagogica.edu.co

4 Universidad Pedagógica Nacional. Bogotá, Colombia. Contacto: lcarmargo@pedagogica.edu.co
} 


\section{Resumo}

Nós descrevemos uma experiência de sala de aula na qual o programa GeoGebraPrim desempenha um papel na introdução da atividade de graduação quarta série demonstrativo, que são convidados para a construção de figuras geométricas, explorando em busca de regularidades e justificar algumas propriedades com base em outro. A experiência é baseada na "atividade demonstração" grupo de pesquisa construção Ensino e Aprendizagem de Geometria da Universidade Nacional Pedagógica e da definição proposta por Stylianides demo (2007).

Palavras-chave: sala de aula, recursos pedagógicos, recursos de computação, software, GeoGebraPrim; aprendizagem processos cognitivos, processos de justificação; atividade demonstração, exploração, conjeturación; justificação.

\section{Contextualización}

Este escrito hace parte de los avances del trabajo de grado "La actividad demostrativa: una mirada desde la educación primaria" que se viene desarrollando en el programa de Maestría en Docencia de la Matemática, de la Universidad Pedagógica Nacional, en la línea de investigación: argumentación y prueba en geometría. La investigación tiene como objetivo principal diseñar, implementar y evaluar una trayectoria de enseñanza que favorezca la actividad demostrativa con niños de grado cuarto de primaria, cuando trabajan en un ambiente de resolución de problemas, usando geometría dinámica. El interés por introducir a estudiantes de temprana edad en prácticas demostrativas no es ni local, ni mucho menos nacional, es un asunto que actualmente hace parte de los temas de investigación en Educación Matemática (Mariotti 2006; Stylianides, 2007).

En la fase experimental de la investigación se usó el programa GeoGebraPrim pues se consideró adecuado para la edad de los niños (8 y 9 años). A diferencia de GeoGebra, este programa permite que los niños vayan construyendo su propia barra de herramientas, a medida que se van introduciendo los objetos y relaciones geométricas en el curso. Además, los puntos y líneas son de mayor tamaño para favorecer la manipulación y la visualización. Al registrar los datos para el trabajo de grado, se pudo apreciar que el uso del programa generó una alta motivación en los niños y ellos se involucraron en procesos como construir, explorar, descubrir, verificar y justificar. Además, el uso del programa para resolver una secuencia de problemas permitió desarrollar los temas previstos para la geometría de cuarto grado, en la institución en donde se llevó a cabo la experiencia, de tal forma que las definiciones y propiedades geométricas se introdujeron a medida que se necesitaban y como respuesta a inquietudes de los estudiantes. En esta ponencia presentamos una síntesis del marco de referencia que fundamenta la experiencia de aula y describimos sus características centrales. Esperamos con ello motivar a otros docentes a usar programas de geometría dinámica en la educación primaria.

\section{Marco conceptual}

En la actualidad, la Educación Matemática tiene como propósito transmitir las bases de la "cultura de los matemáticos", más allá de transmitir información. Esto implica que los maestros se comprometan con el desarrollo de propuestas curriculares que no estén centradas en aprender contenidos (Artigue, 2002). El software GeoGebraPrim es un posibilitador que, acompañado de un diseño cuidadoso de actividades, permite nuevas opciones curriculares centradas en actividades matemáticas como resolver problemas, hacer construcciones geométricas, descubrir propiedades y justificarlas.

En la experiencia de aula motivo de esta ponencia, se optó por usar el programa GeoGebraPrim en una secuencia de problemas fundamentada en el 
constructo actividad demostrativa sugerido por el grupo $€ \bigotimes$ G como una alternativa para organizar las clases, que impulsa la exploración de construcciones geométricas, el descubrimiento y verificación de propiedades y la justificación de las mismas. En la medida en que los niños logren justificar algunas propiedades usando hechos geométricos y no recurran a la percepción visual, comienzan un proceso de acercamiento al mundo teórico de la geometría, a temprana edad. En dicho proceso, las figuras geométricas dejan de verse como ilustraciones de objetos físicos y sus características empiezan a verse como propiedades geométricas más que como atributos estéticos.

Después de que los niños descubren una propiedad geométrica, pueden ser guiados por el maestro a elaborar una demostración, entendida como la define Stylianides (2007), como un argumento matemático, es decir, una secuencia conectada de afirmaciones a favor de un enunciado matemático que provienen de un sistema de conocimientos en el que los estudiantes: usan hechos geométricos que son aceptados por la comunidad de clase, usan formas de razonamiento que son válidas, conocidas y al alcance de la comunidad de clase, y usan formas de comunicarse acordadas por el grupo.

\section{Descripción de la experiencia}

La experiencia de aula se desarrolló con 30 estudiantes de cuarto grado, en las clases de geometría, programadas en los dos últimos meses de año escolar. En total, se llevaron a cabo 9 sesiones de clase de 100 minutos aproximadamente, que se realizaron en la sala de informática de la institución. Los niños trabajaban en grupos de dos o tres personas, usando el programa GeoGebraPrim para resolver ocho problemas de geometría plana, propuestos por la profesora, y elaboraban un reporte de su trabajo usando la opción 'texto' del software. Después del trabajo en grupo, se impulsaba una socialización en la que los niños presentaban su trabajo y la discusión se centraba en la justificación de las propiedades encontradas. A continuación se mencionan cuatro de los problemas propuestos y se ilustra la actividad de los niños con ejemplos de la producción de algunos grupos.

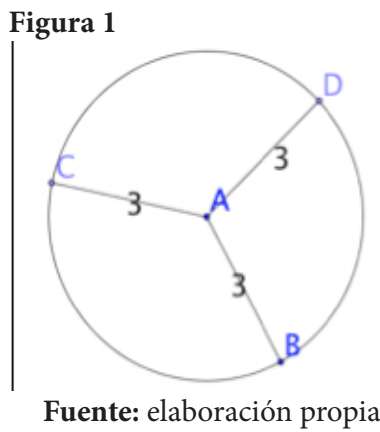

En el problema 1, se les pidió a los estudiantes construir una circunferencia con centro en $\mathrm{A}$, ubicar tres puntos $\mathrm{B}, \mathrm{C}$ y $\mathrm{D}$ en ella, trazar los segmentos $\mathbb{\nabla}$ $\bigotimes$ y descubrir una propiedad de dichos segmentos. Luego de hacer la construcción, la mayoría de grupos conjeturaron que los segmentos tienen la misma medida; usan las herramientas 'longitud' y 'arrastre' (Fig. 1) para verificar la conjetura. El problema sirve para introducir las definiciones de radio y de congruencia de segmentos así como la propiedad: los radios de una circunferencia son congruentes.

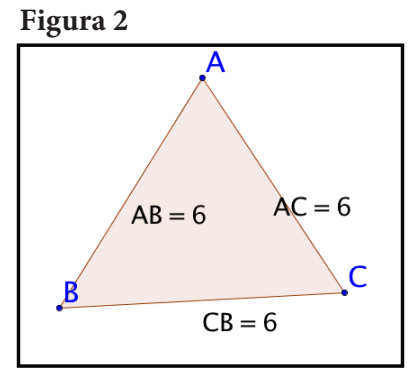

Fuente: elaboración propia

En el problema 2 se les pidió a los estudiantes construir un triángulo equilátero. Inicialmente, la mayoría de grupos construyó un triángulo $\mathrm{ABC}$ con la herramienta 'polígono', con la herramienta 'longitud' midieron sus lados $\mathrm{AB}, \mathrm{BC}$ y $\mathrm{AC}$, arrastraron los vértices del triángulo hasta que en el pantalla se mostrará que todos los lados tenían igual medida. En algunos casos, a pesar de obtener el mismo valor en las longitudes, el triángulo no lucía equilátero (Fig. 2).

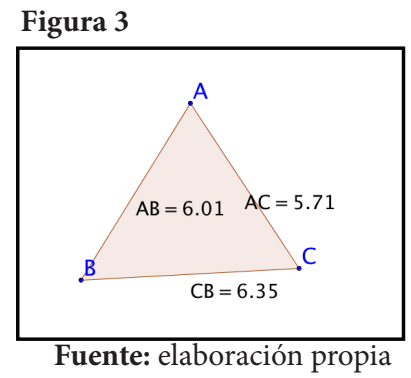


Esto se debe a que el programa GeoGebraPrim redondea las medidas para mostrarlas en números naturales ya que este el dominio numérico más usado en primaria. La profesora les explicó cómo hacer para que el programa mostrara las medidas con dos cifras decimales y así pudieron obtener un triángulo con apariencia de triángulo equilátero. (Fig. 3). Sin embargo, como la congruencia de los lados no era invariante al arrastre, la profesora invitó a los estudiantes a construir el triángulo a partir del $\bigotimes$ y dos circunferencias de radio BC (Fig. 4). La tarea se convirtió en oportunidad para diferenciar entre dibujos y figuras, considerando las últimas como aquellas que conservan las propiedades geométricas que las caracterizan, aunque varíen otras. El problema sirvió para introducir la definición de triángulo equilátero y justificar, con base en la propiedad descubierta en el problema uno, por qué los segmentos $\nabla \nabla \nabla$ de la figura 4 son congruentes.

Figura 4

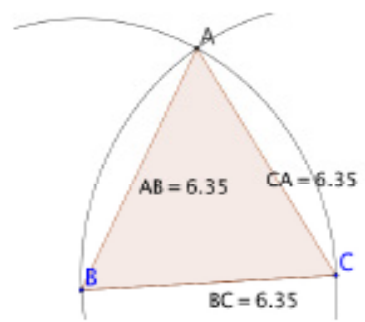

Fuente: elaboración propia

En el problema 5 los niños debían construir dos segmentos $\nabla$ Qque fueran congruentes y que se bisecarán en un punto $\mathrm{C}$, luego debían unir los puntos $\mathrm{A}, \mathrm{B}, \mathrm{D}$ y $\mathrm{F}$ en ese orden, y descubrir qué cuadrilátero podría ser. Para resolver el problema, un grupo de estudiantes construyó $\bigotimes$ y con la herramienta "punto medio" halló el punto C; luego, usó la herramienta "compás" para construir un $\nabla y$ halló el punto medio de este segmento. Luego, los niños arrastraron el $\otimes$ hasta que en la pantalla se observará que ambos segmentos se bisecaban en el punto C (Fig. 5); con la herramienta "polígono" trazaron el cuadrilátero ABDF y con la herramienta "ángulo" midieron los ángulos del cuadrilátero y se dieron cuenta que las medidas se aproximaban a 90 grados. Los estudiantes descubrieron que el cuadrilátero podía ser un rectángulo, y conjeturaron que si en un cuadrilátero las diagonales son congruentes y se bisecan, el cuadrilátero es rectángulo.

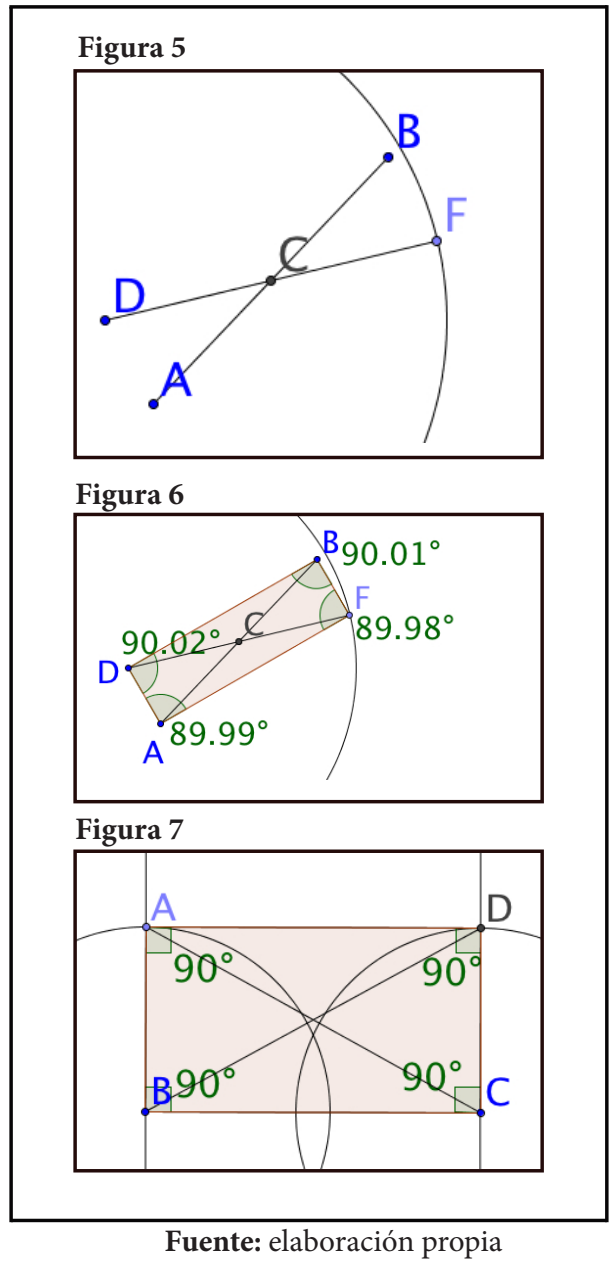

La profesora aprovechó la oportunidad para introducir la construcción del rectángulo, a partir de un $\bigotimes$, rectas perpendiculares por B y C y circunferencias con centro en $\mathrm{B}$ y $\mathrm{C}$ y radio $\mathrm{BA}$.

Con la figura construida usando las propiedades del rectángulo, se introdujo su definición y la definición de rectas perpendiculares. Los estudiantes analizaron propiedades de las diagonales, lo que les permitió corroborar la propiedad anterior.

En el problema 7 se pidió a los niños construir un $\triangle \mathrm{ABC}$, determinar el punto medio $\mathrm{D}$ del lado $\mathrm{AB}$, trazar el $\nabla$ y estudiar en qué casos los $\nabla \nabla \nabla$ son congruentes. Los niños descubrieron que esta propiedad se cumple cuando el triángulo es rectángulo en C. Además de introducir la definición de 
punto medio, se definió triángulo rectángulo. En el problema 8 se pidió a los estudiantes construir una circunferencia con centro $\mathrm{O}$, un diámetro $\mathrm{AB}$, un punto $\mathrm{D}$ en ella y el $\triangle \mathrm{ABD}$. Luego, arrastrar el vértice $\mathrm{D}$, investigar una propiedad del triángulo y justificarla. Los estudiantes descubrieron que el $\triangle \mathrm{ABC}$ era rectángulo. En varios grupos de estudiantes los niños propusieron dos justificaciones de la propiedad a partir de las propiedades descubiertas en los problemas 5 y 7 . Como los niños usan hechos geométricos que se han estudiado en la clase, usan el lenguaje geométrico aprendido y esbozan un razonamiento deductivo incipiente, se puede afirmar que han iniciado un camino hacia el mundo teórico de la geometría logrando hacer una demostración.

\section{Logros y dificultades}

Se considera que el programa GeoGebraPrim resulta ser motivante para los estudiantes de grado cuarto de primaria, lo que permite que los estudiantes se interesen mucho más por los procesos mismos de la actividad demostrativa. Sin embargo, se ve necesario que el profesor sea mediador entre la experiencia empírica que se adquiere con el programa y la teoría geométrica.

\section{Reflexión final}

Para que el programa GeoGebraPrim sea un instrumento para introducir a los niños en la actividad demostrativa, deben proponérseles a los estudiantes tareas de resolución de problemas abiertos que de acuerdo con Fulvia Furinghetti et al. (2000), se caracterizan por ser enunciados cortos que sean fácilmente entendibles para que los estudiantes puedan iniciar el proceso de solución. Los enunciados no sugieren el método de solución pero crean situaciones que estimulan la conjeturación, y los problemas deben proveer que los estudiantes puedan crear ejemplos y contraejemplos.
El programa GeoGebraPrim mediado por la teoría geométrica, puede llegar a convertirse en un micromundo, (Baccaglini, Mariotti, 2009), donde los "objetos" (punto, recta, etc.) se pueden manipular y se constituyen en objetos de experimentación. En ese sentido, se convierten en mediadores entre el saber matemático y el saber que está siendo construido por el niño.

\section{Referencias}

Artigue M. (2002). Learning mathematics in a CAS environment: the genesis of a reflection about instrumentation and the dialectis between technical and conceptual work. International Journal of Computers for Mathematics Learning, 7, 245-274.

Bartolini Bussi, M.G. y Mariotti, M.A. (2008). Semiotic mediation in the mathematics classroom: Artifacts and signs after a Vygotskian perspective. En L. English, M.G. Bartolini Bussi, G. Jones, R. Lesh y D. Tirosh (Eds.), Handbook of international research in mathematics education, segunda edición revisada. Mahwah: Lawrence Erlbaum.

Baccaglinni, A \&Mariotti, M (2010). Conjeturing and proving in dynamic geometry: the elaboration of some research hypoteses. En Proceedings of CERME 6, January 28th-February 1st 2009, Lyon France.

Mariotti, M. A. (2006). Proof and proving in mathematics education. In A. Gutiérrez \& P. Boero (Eds.), Handbook of research on the psychology of mathematics education: Past, present and future (pp. 173-204). Rotterdam / Taipei: Sense Publishers.

Stylianides, A.J. (2007). Proof and proving in school mathematics. Journal for Research in Mathematics Education, 38, 289-321. 